\section{LA PLANTA DE ARENAS DEL IETCC}

\author{
Manuel Chinchilla, \\ Titulado Superior Especializado (IETCc) \\ $071-13$
}

\section{PREAMBULO}

Las características mecánicas, químicas y fisicoquímicas de los cementos exigen, para su determinación y evaluación, la necesidad de utilizar métodos de ensayo que han de llevarse a cabo con probetas construidas de morteros específicos.

Para la preparación de estos morteros es obligado seguir unos procesos prefijados de amasado y elaboración, así como es imprescindible que los componentes cumplan unos condicionamientos sumamente estrictos en cuanto a cantidades, calidad, proporciones y composición, ya que, al pretender obtenerse de la diferenciación de resultados unos parámetros que permitan deducir las cualidades del «elemento-cemento», es ineludible que todos los demás factores y componentes no introduzcan ni ocasionen interferencias, ni variables que puedan falsear las deducciones, o inducir a consecuencias erróneas.

En lo concerniente al árido, que debe ser utilizado, se exige de éste un riguroso condicionamiento, no sólo en lo concerniente a su cantidad exacta, sino en todo lo referente a sus características de composición química (alto contenido en sílice, no inferior al $98 \%$ ), coeficiente de forma, densidad, aparente y específica, contenido de humedad y curva granulométrica.

Su delimitada tipología lleva consigo que esta clase de arenas sólo pueda ser preparada a partir de materiales procedentes de un número muy escaso de yacimientos en el mundo.

En España, en una determinada zona de Segovia, se encuentra un tipo de arena que puede ser considerada, internacionalmente, como una de las más adecuadas, en lo que concierne a sus cualidades primarias.
El Instituto Eduardo Torroja - pionero en este campo, desde el año 1957 - ha llegado a disponer actualmente de una. Planta de Preparación de Arenas, que si todavía está lejos de reunir las posibilidades óptimas, es la que suministra, satisfactoriamente, arenas especiales, normalizadas y calibradas, para este tipo de ensayos y otros trabajos de Laboratorio, no sólo a la Industria Nacional, sino a bastantes países extranjeros, que si no representan un mayor número y volumen de consumo, se debe únicamente a falta de una mayor capacidad de producción de la Planta, pero no por existir falta de demanda, ni posibilidades de creación de nuevos mercados.

Las clases de arena, actualmente producidas, son:

- Arena Normalizada.-Según las especificaciones de la Norma del «B.O.E.»n. 22 de 26 de enero de 1960 , y PRC-75.

- Arena Patrón.-Similar en cuanto a sus características a la Arena Normalizada, pero constituida por una granulometría con menores márgenes de tolerancia.

ZONA GRANULOMETRICA ADMISIBLE PARA LA ARENA NORMALIZADA

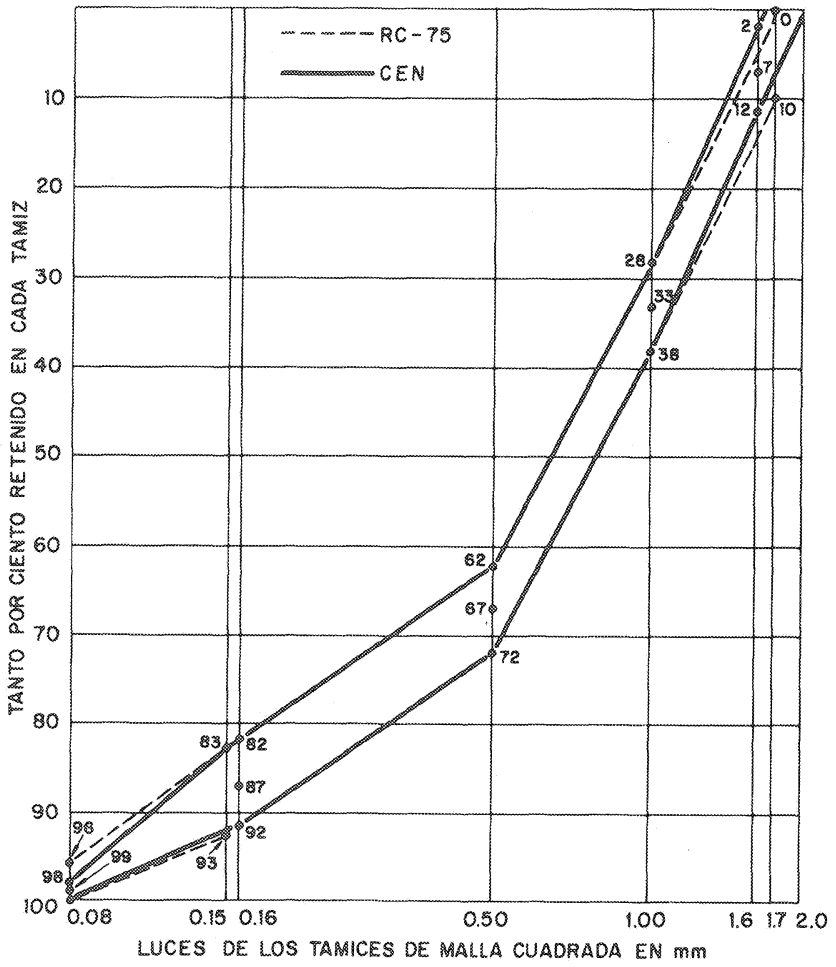


ARENA NORMALIZADA

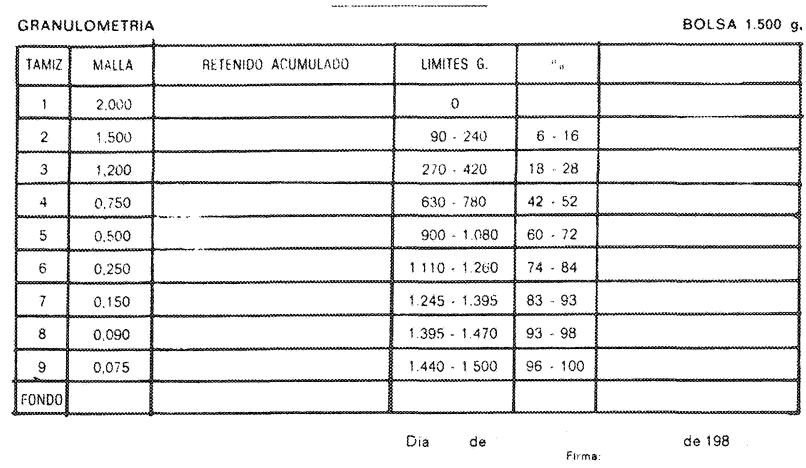

de de 198 ARENA PATRON
Horas
GRANULOMETRIA
\begin{tabular}{|c|c|c|c|c|c|c|c|}
\hline TAMIZ & MALLA & \multicolumn{3}{|c|}{ RETENIDOS ACUMULADOS } & LMITES 6 & $\%$ \\
\hline 1 & & & & & & 0 & \\
\hline 2 & & & & & & $150 \cdot 240$ & $10 \cdot 16$ \\
\hline 3 & & & & & & $330 \cdot 420$ & $22 \cdot 28$ \\
\hline 4 & & & & & & $660 \cdot 780$ & $44 \cdot 52$ \\
\hline 5 & & & & & & $960 \cdot 1.080$ & $64 \cdot 72$ \\
\hline 6 & & & & & & $1.114 \cdot 1.260$ & $76 \cdot 84$ \\
\hline 7 & & & & & & $1.270 \cdot 1.395$ & $85 \cdot 93$ \\
\hline 8 & & & & & & $1.410 \cdot 1.470$ & $94 \cdot 98$ \\
\hline 9 & & & & & & $1.440 \cdot 1.500$ & $96 \cdot 100$ \\
\hline FONDO & & & & & & & \\
\hline
\end{tabular}

Su campo de aplicación es el de la arena normalizada, además de ser empleada, por sus posibilidades, como testigo «inter-laboratorios», para estudios comparativos de homogeneización de resultados, estudio de procesos, tarados de prensas, y calibraciones comparativas.

- Arenas calibradas.-En sus diferentes tamaños son empleadas para trabajos de determinación de densidades en terrenos in situ, y en todos aquellos campos en que se necesite un material inerte, de composición, densidad y granulometrias constantes.

- Arenas tipo Ottawa.-Actualmente en preparación, en previsión de la aprobación y desarrollo del Programa de Investigación, que someramente se expone al final del artículo.

\section{DESCRIPCION DE LA PLANTA}

\section{a.-Sección de clasificación}

La arena, procedente del yacimiento, seca y preclasificada en cinco granulometrías diferentes, es recogida en una tolva de recepción de primarios.

Desde allí y mediante un sistema de transporte por vacío es trasladada, para su acopio, al correspondiente silo de almacenamiento.

Cada uno de los cuatro silos de la batería, en forma independiente y mediante regulación semi-automática, va dejando caer, a la tolva de homogeneización, la proporción correspondiente de material al todo-uno que se quiere recomponer. De esta forma, en la tolva queda constituida una granulometría que corresponde, "grosso-modo», con la de la arena normalizada, patrón o calibrada que quiere producirse.

Desde la tolva de homogeneización, el material recompuesto es trasladado, también, mediante un sissistema de aspiración por vacío a la torre de clasificación, que está compuesta por dos cribadoras, en serie, con ocho tamices de doble paso.

La estación clasificadora efectúa las pertinentes separaciones del todo-uno recibido para su pase, por gravedad, a cada una de las tolvas de la dosificadora $\mathrm{o}$, en su caso, a los correspondientes tolvines de arena calibrada.

\section{b.-Dosificación y envasado}

La máquina dosificadora va recibiendo, en cada una de sus tolvas, el correspondiente tamaño separado en la torre de clasificación, y mediante control electrónico dosifica las proporciones correspondientes a la curva granulométrica programada, efectuando el llenado de las bolsas con un peso total de $1.500 \mathrm{~g}$, con un error total de $\pm 1 \mathrm{~g}$.

El circuito alimentador a las tolvas de la dosificadora está proyectado de forma que, el

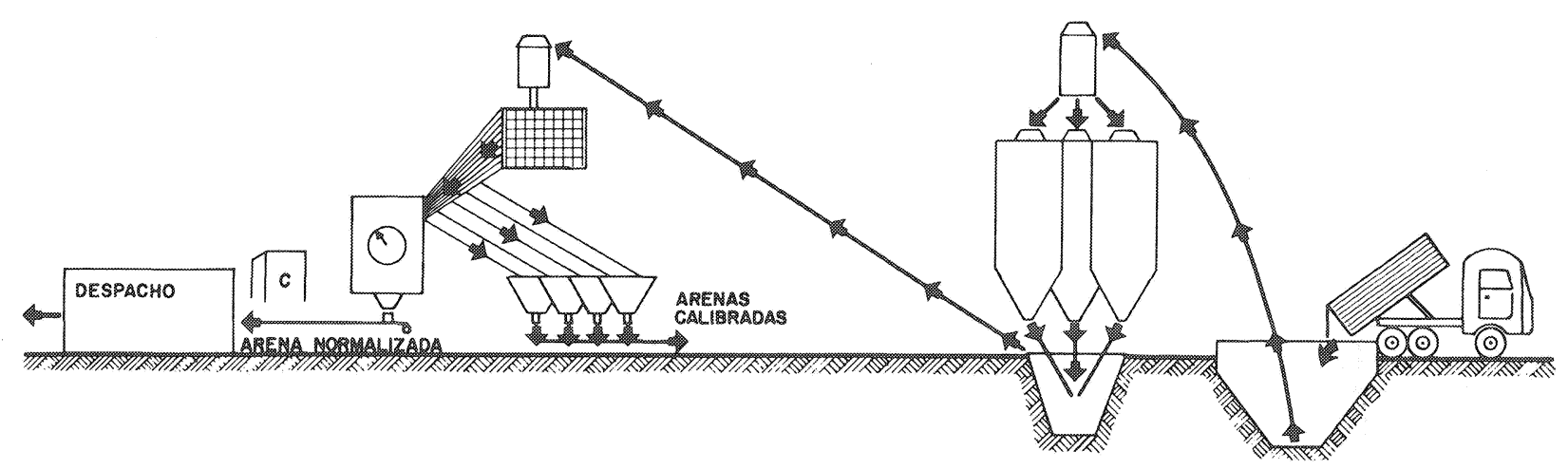


posible exceso de un determinado tamaño de material cribado, pasa para su posterior aprovechamiento al oportuno tolvín de arena calibrada.

Una vez ha sido envasada la carga en su bolsa de plástico, éstas son pasadas, una a una, a la soldadora térmica. La experiencia ha demostrado como más conveniente efectuar esta operación en forma independiente y unitaria, lo cual permite un control exhaustivo.

Después de esta operación, el material ha quedado absolutamente seco y con la granulometría correcta $\mathrm{y}$ en bolsas herméticamente selladas y dispuestas para su posterior introducción en cajas de cartón, con capacidad para diez bolsas, cada una de ellas.

\section{c.-Laboratorio de control}

Se efectúan en él las verificaciones oportunas para la recepción del material, con objeto de comprobar la ausencia de impurezas y contaminaciones en la arena, recibida del yacimiento; así como que la misma se ajusta al contenido en sílice, forma y tamaño a lo especificado.

Como control de la calidad de producción, mediante tamices vibratorios, se efectúan análisis por muestreo de las bolsas ya preparadas, con objeto de que quede garantizado que se encuentran dentro de las tolerancias permisibles. Se aprovecha este seguimiento para llevar un estudio previo de las tendencias granulométricas, lo que permite introducir a priori correcciones en la programación de la dosificadora.

\section{d.-Observaciones generales}

Dadas las especiales características del material manejado, su índice de peligrosidad - silicosissu alto poder abrasivo, y la extremada precisión exigida, es ineludible - y la práctica así lo ha corroborado- que es necesario que este tipo de instalaciones, y las máquinas empleadas, estén diseñados y construidos exprofeso para el manejo de esta clase de material. Todos los transportes han de hacerse en conductos cerrados y mediante sistemas de aspiración por vacío, con reguladores de apertura y cierre anulares con válvulas de diafragma. Las condiciones de seguridad e higiene del trabajo deben ser rigurosamente observadas, y se deberán sobredimensionar y multiplicar los puntos de aspiración y ventilación, que impidan la contaminación ambiental por polvo.

\section{e.-Datos de producción}

En la actualidad la instalación está funcionando a su capacidad máxima de rendimiento, el cual, si

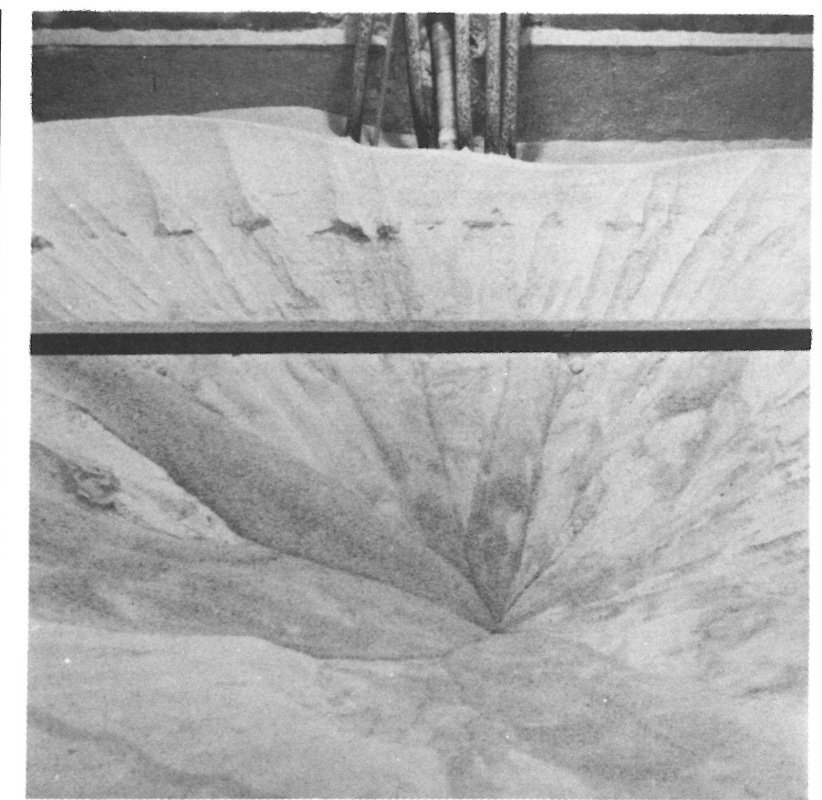

Tolva de primarios.

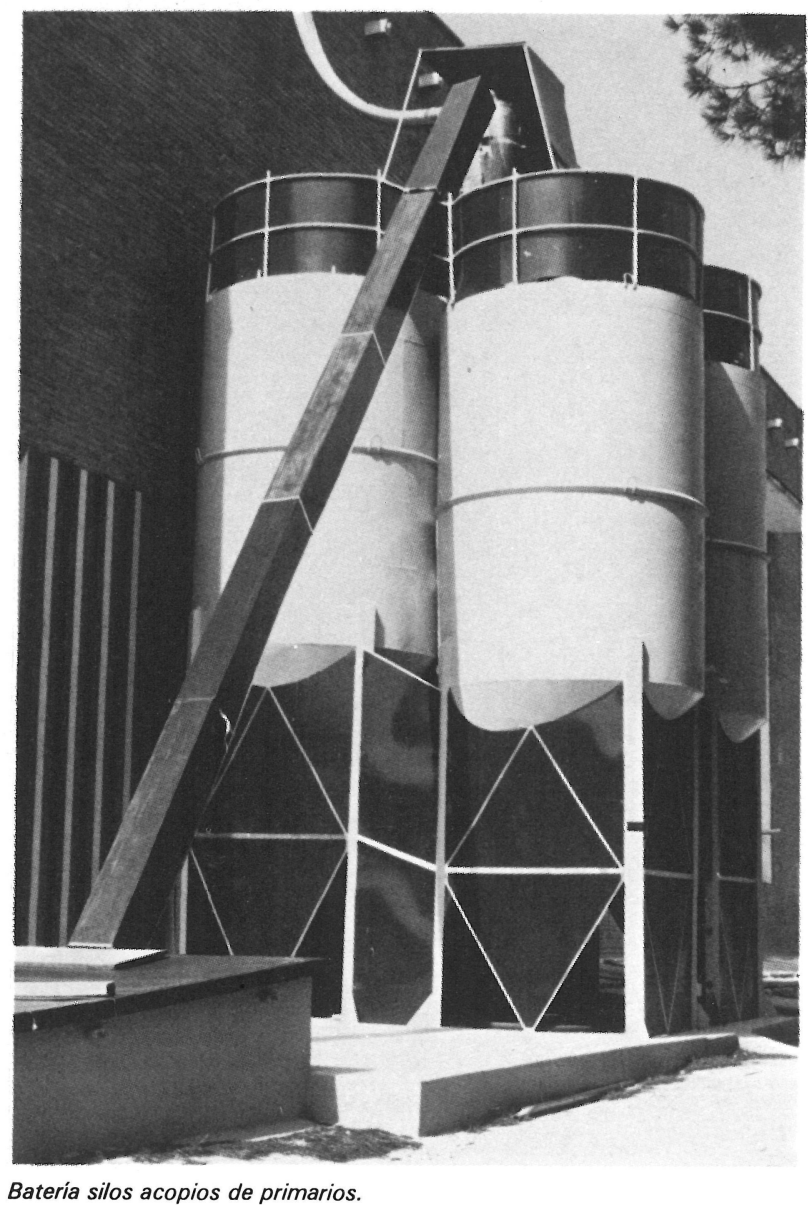


bien es, por el momento, suficiente para la demanda del consumo nacional, no permite atender debidamente, las demandas existentes del exterior.

El volumen de producción es:

- 2.100 cajas mensuales, con 21.000 bolsas de 1.500 g, de Arena Normalizada.

- 200 cajas mensuales de Arena Patrón.

- 300 sacos de $25 \mathrm{~kg}$ de arenas calibradas, al mes.

- $3.500 \mathrm{~kg}$. de arenas de rechazo, para morteros especiales, cultivos hidropónicos, etc.

\section{f.-Otras consideraciones}

Recientemente, Japón, Argentina, Venezuela y Perú, han enviado técnicos especiales para visitar la instalación de arenas del IETcc, con vistas al posible montaje de instalaciones similares en sus respectivos países.

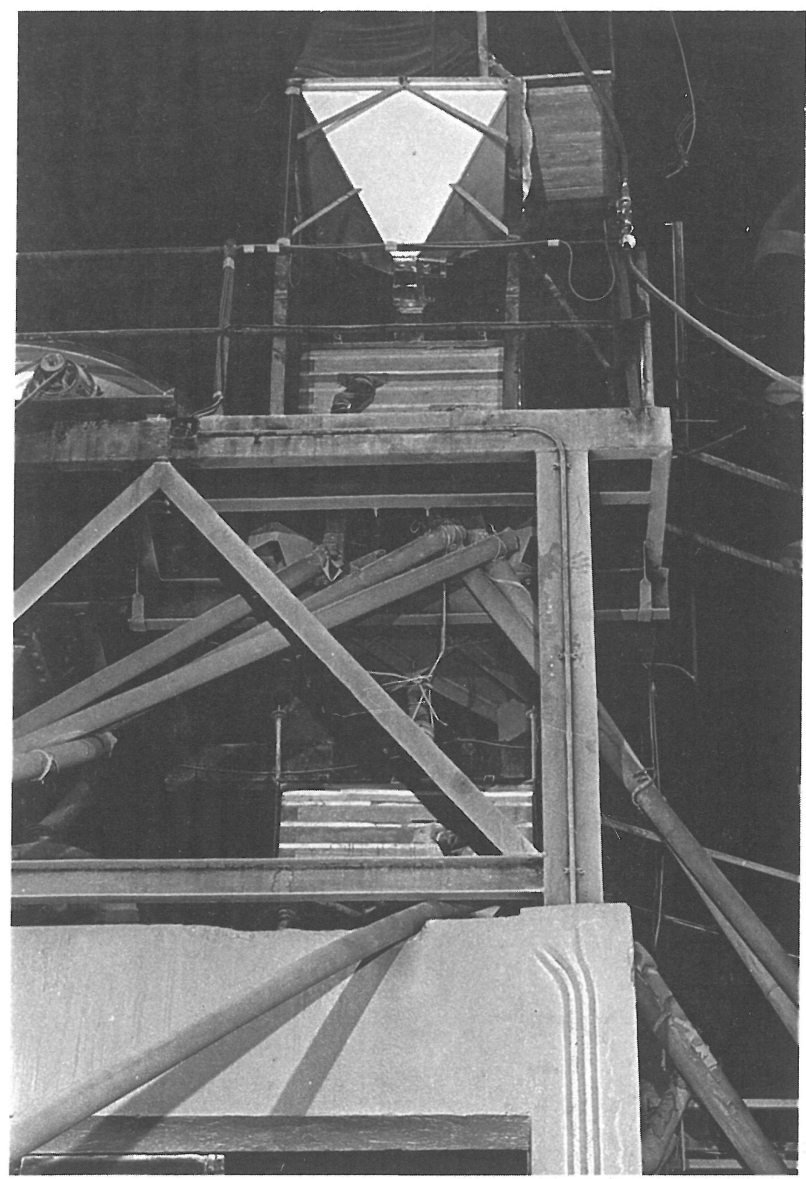

Torre de clasificación.

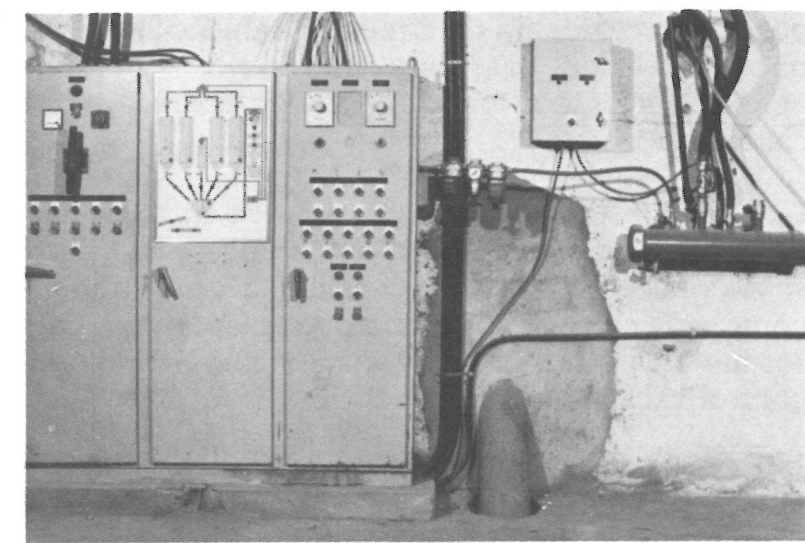

Cuadro regulación automática descarga-silos.

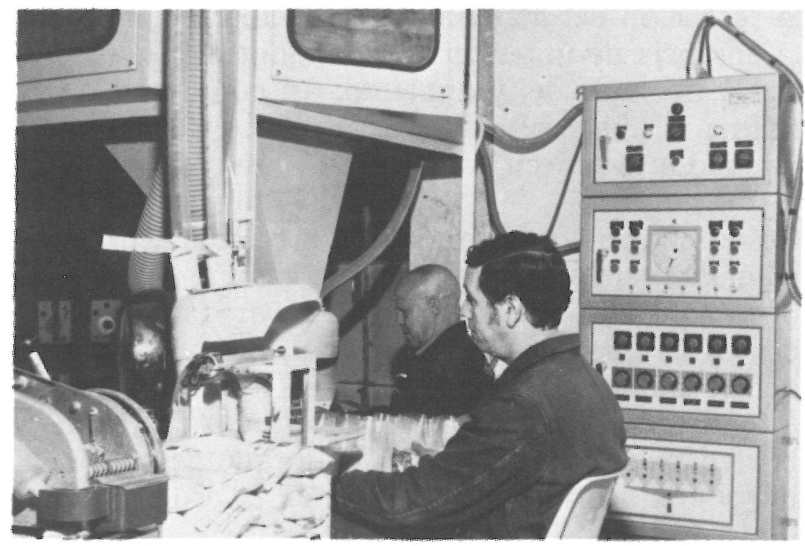

Dosificación y envasado.

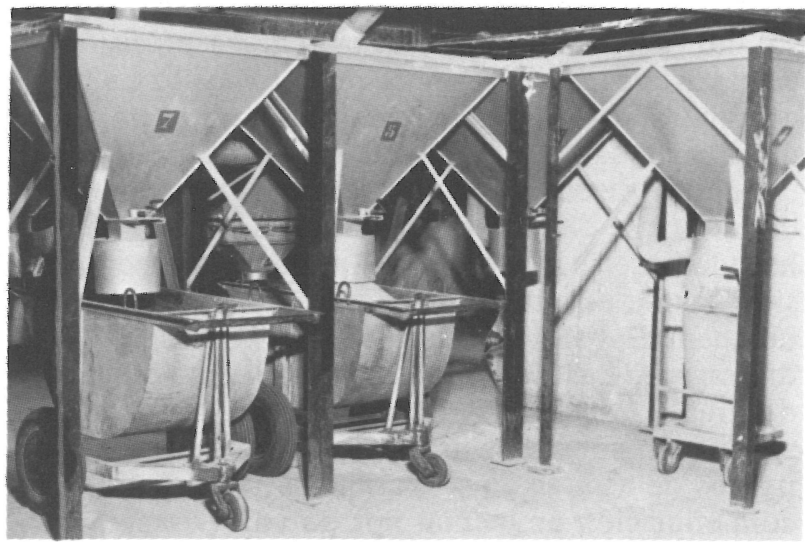

Tolvines de arena calibrada. 


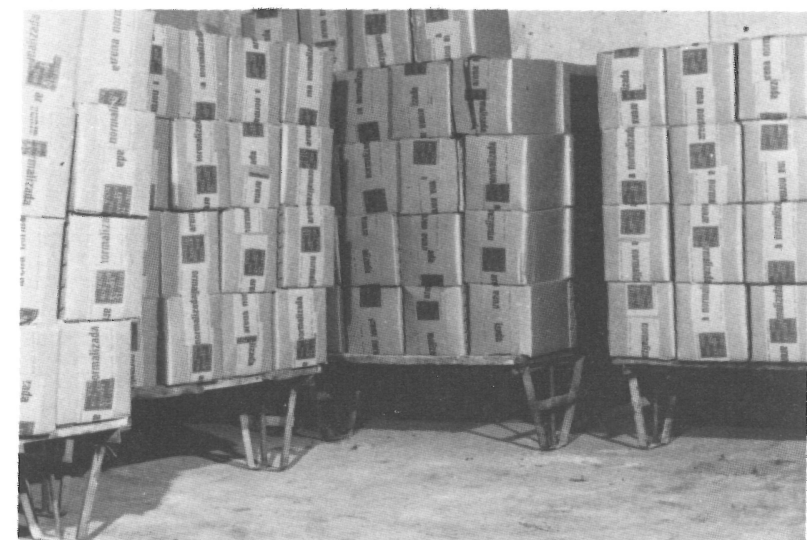

Arena normalizada preparada para su envío. clasificada tipo Ottawa, que es la que amparan las antedichas normas ASTM.

Ante esta problemática, el IETcc tiene solicitada la aprobación de un programa de investigación, cuya meta es llegar a conseguir partiendo de la arena española - una arena «tipo Ottawa»-que posibilite la elaboración de una norma española UNE, que sea aceptada como norma CEN, y equiparada a nivel internacional con las correspondientes ASTM.

Con ello sería posible sustituir la arena de Ottawa por una arena española "Tipo Ottawa», lo cual representaría las siguientes ventajas:

- facilidad tecnológica para el control de calidad de la fabricación de cemento español, con destino a la exportación, para acomodarse a las condiciones imperantes en parte del mercado extranjero. Dicho control podría ser llevado a cabo, sin necesidad de efectuar importaciones de arena Ottawa, con el subsiguiente ahorro de divisas;

- posibilidad de complementar las exportaciones de Arena Normalizada actual, con Arenas españolas 2 «Tipo Ottawa», especialmente para aquellos países que emplean en la actualidad cemento español, o disponen de fábricas con tecnología española y que, sin embargo, actualmente han de continuar bajo la obligatoriedad de la clase de arena impuesta en las normas ASTM. Este libro, el primero en lengua castellana sobre resinas epoxi aplicadas a la construcción, está dirigido a arqui-
tectos, ingenieros, constructores y aplicadores. En él, sobre una reducida base teórica imprescindible, se asienta toda una extensa gama de aplicaciones de gran interés.

El autor trabaja desde hace muchos años en el campo de la investigación, especialmente en el estudio de refuerzos y reparaciones estructurales realizados con resinas epoxi.

Con un lenguaje sencillo se tocan todos los problemas que pueden presentarse en la construcción y en los que la solución puede radicar en el correcto empleo de las resinas epoxi.

Se estudian los componentes de las formulaciones epoxi, sus propiedades fisicas y químicas, y aplicaciones, deteniéndose, detalladamente, en las siguientes:

Unión de hormigón fresco a hormigón endurecido. - Unión de hormigones entre sí.-Inyecciones de fisuras y grietas. - Unión de acero a hormigón. - Barnices y pinturas. - Las combinaciones brea-epoxi. - Revestimientos de depósitos alimenticios. - Sellado de superficies cerámicas. - Protección de tubos. - Los suelos epoxi en sus diferentes variantes. - Terrazo epoxi. - Reparación de baches. - Reparación de desperfectos en estructuras. - Reparación de carreteras de hormigón. - Juntas elásticas. - Guardacantos de tableros de puentes. - Refuerzos de pilares,
vigas, forjados y zapatas, etc. - Consolidación de suelos. - Anclajes. - Protección de aceros en pretensado.

Se termina con unos capitulos dedicados a la limpieza y preparación de las superficies según los materiales a unir; al control del estado superficial de éstos; a las condiciones de temperatura de aplicación; limpieza de los útiles de trabajo; precauciones en el manejo de los sistemas; almacenaje, mezcla y manejo de las formulaciones epoxi y
métodos de ensayo de sistemas y aplicaciones epoxidicas.

Un volumen encuadernado en cartoné plastificado con lomo de tela, de $17 \times 24 \mathrm{~cm}$, compuesto de 334 páginas y 158 figuras y fotografias.

Madrid, 1981.

Precios: España, 1.700 ptas.; extranjero, \$ USA 34.00. 